\title{
A somatic PIK3CA p.H1047L mutation in a Thai patient with isolated macrodactyly: a case report
}

\author{
Wandee Udomchaiprasertkul ${ }^{1,2,3,4}$, Pravit Kitidumrongsook ${ }^{5}$, Kanya Suphapeetiporn ${ }^{1,2, *}$, \\ Vorasuk Shotelersuk ${ }^{1,2}$
}

Abstract

Background: Macrodactyly is a rare congenital deformity characterized by gigantism of all structures of the digits. Previous reports showed that the mosaic PIK3CA variants were associated with overgrowth syndromes including macrodactyly.

Objectives: To determine the genetic alteration in a Thai patient with enlarged soft tissue of the left middle and left fourth fingers with abnormal enlarged phalanges.

Method: A nerve and a skin piece were separated from a therapeutically surgically removed part of the enlarged digit. Skin fibroblasts were cultured from the removed skin piece. DNA was isolated from the nerve tissue, cultured skin fibroblasts, and peripheral blood leukocytes. Polymerase chain reaction (PCR) followed by Sanger sequencing of PIK3CA was performed.

Results: Mutation analysis revealed the c.3140A >T (p.(H1047L)) variant of PIK3CA in the nerve tissue and the cultured dermal fibroblasts but not in leukocytes from the peripheral blood.

Conclusion: The somatic c.3140A $>$ T (p. $(\mathrm{H} 1047 \mathrm{~L})$ ) variant of PIK3CA was found in a Thai patient with isolated macrodactyly, the same as those previously identified in other populations.

Keywords: macrodactyly; mutation; $P I K 3 C A$

Macrodactyly refers to a rare congenital deformity characterized by digital gigantism of all structures including subcutaneous fat, tendons, nerves, vessels, skin, and nails [1]. The prevalence of macrodactyly is about 0.08 per 10,000 live births [2].
Macrodactyly can be inherited when it occurs with a syndrome, but isolated macrodactyly is usually sporadic [3, 4]. Macrodactyly of the hand is more frequently than the foot and usually involved more than one digit. The enlarged digits may grow

\footnotetext{
*Correspondence to: Kanya Suphapeetiporn, Department of Pediatrics, Faculty of Medicine, Chulalongkorn University, Bangkok 10330, Thailand, e-mail: kanya.su@chula.ac.th

'Center of Excellence for Medical Genomics, Department of Pediatrics, Faculty of Medicine, Chulalongkorn University, Bangkok 10330, Thailand 2Excellence Center for Genomics and Precision Medicine, King Chulalongkorn Memorial Hospital, the Thai Red Cross Society, Bangkok 10330, Thailand

${ }^{3}$ Interdisciplinary Program of Biomedical Sciences, Graduate School, Chulalongkorn University, Bangkok 10330, Thailand ${ }^{4}$ Molecular Biology and Genomic Research Laboratory, Division of Research and International Relations, HRH Princess Chulabhorn College of Medical Science, Chulabhorn Royal Academy, Bangkok 10900, Thailand

${ }^{5}$ Department of Orthopedics, Faculty of Medicine, Chiang Mai University, Chiang Mai 50200, Thailand
}

כ Open Access. @ 2019 Wandee Udomchaiprasertkul et al., published by Sciendo. (c) BY-NC-ND This work is licensed under the Creative Commons Attribution NonCommercial-NoDerivatives 4.0 License. 
either in static or progressive condition $[2,5]$. Treatments of macrodactyly comprise soft tissue debulking, nerve stripping, epiphysiodesis, osteotomies, and phalangectomy [2].

Mutations in the PIK3CA gene have been previously described as the cause of isolated macrodactyly in Caucasian patients. The mutations were detected only in the affected tissues but not in peripheral blood [6]. Moreover, the mosaic mutations in PIK3CA were found to be correlated with several overgrowth disorders. Therefore, the umbrella term of PIK3CA-related overgrowth spectrum (PROS) was nominated to designate the broad range of clinical symptoms including isolated macrodactyly, fibroadipose overgrowth (FAO), hemihyperplasia multiple lipomatosis (HHML), congenital lipomatous overgrowth, vascular malformations, epidermal nevi, scoliosis/skeletal and spinal (CLOVES) syndrome, and related megalencephaly conditions $[4,7,8]$.

We identified a Thai patient with diffuse enlarged left middle and left fourth fingers. As pathogenic variants in PIK3CA have been reported as the cause of this symptom [6], we aimed to determine the genetic alteration in our Thai patient with macrodactyly. The mutation could be either a germline or somatic mutation. Causative germline mutations of a genetic disorder can have different frequencies in various populations.

\section{Materials and methods}

\section{Patient}

The present case report was approved by the Institutional Review Board, Faculty of Medicine, Chulalongkorn University (certificate of approval no. 365/60). The patient's guardian has provided the written consent for the publication of this case report and any accompanying information and images.

Tissue specimens were obtained during surgical procedures performed for the treatment of macrodactyly. A 5-year-old girl with enlarged left middle and fourth fingers was referred for further treatment at King Chulalongkorn Memorial Hospital (Figure 1).

\section{Cell culture}

Skin fibroblasts from the removed affected finger were cultured in Dulbecco's modified Eagle medium (DMEM) (Hyclone, GE Healthcare) supplemented with $10 \%$ fetal bovine serum (Hyclone, GE Healthcare) and 1\% penicillin/streptomycin (Hyclone, GE Healthcare). The morphology of cultured cells

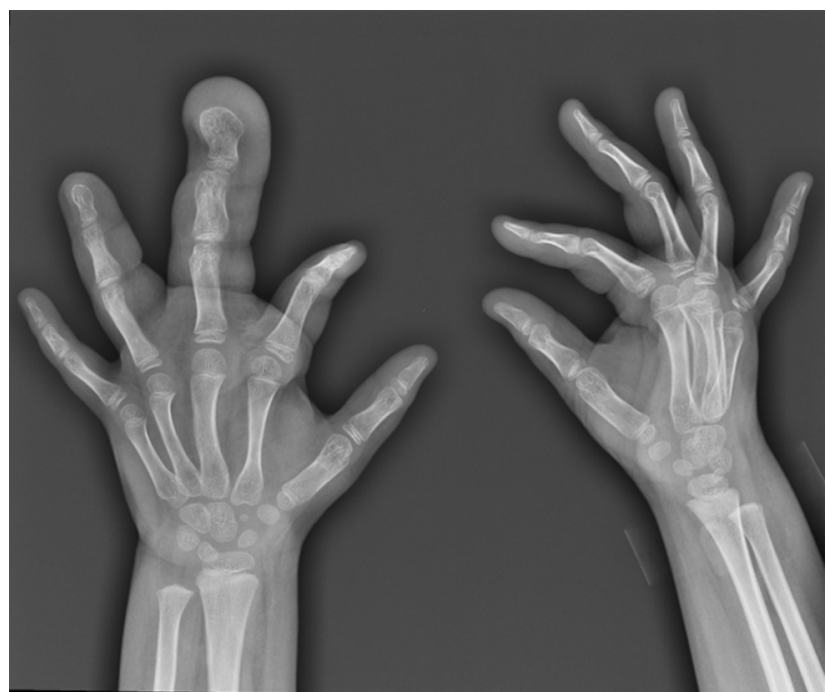

Figure 1. Clinical and radiologic features of the patient. The radiographs show enlarged middle and fourth fingers of the patient's left hand

was consistent with fibroblasts. The cultured dermal fibroblasts were collected for DNA extraction.

\section{Polymerase chain reaction and Sanger sequencing}

DNA was extracted from cultured dermal fibroblasts and affected nerve tissue using the QIAamp according to the manufacturer's instruction (Qiagen). Genomic DNA was separated from leukocytes in peripheral blood using a Puregene blood kit (Qiagen). PIK3CA exon 21 was amplified using the following primers: the forward primer 5'-GTCTACGAAAGCCTCTCTAA-3' and the reverse primer 5'-GCTGTAAATTCTAAGC TGTTC-3'. The polymerase chain reaction (PCR) products were treated with Exo-Sap-IT (Affymetrix) followed by Sanger sequencing. Chromatograms were analyzed using Chromas.

\section{Results}

The patient was evaluated by physical examination and X-ray imaging, which showed diffuse enlarged soft tissue of left middle and left fourth fingers with abnormal enlarged phalanges (Figure 1). The patient did not have obvious asymmetry of other parts of the body. Initially, the patient underwent the surgical correction to reduce the bulk of left middle finger with fusion of distal interphalangeal (DIP) and proximal interphalangeal (PIP) joints. Physical examination and X-ray imaging 1 year after the surgery displayed re-enlargement of the left middle finger (Figure 2). Fatty tissue debulking, corrective and osteotomy of the DIP joint, and fixation by K-wire were performed in the left 


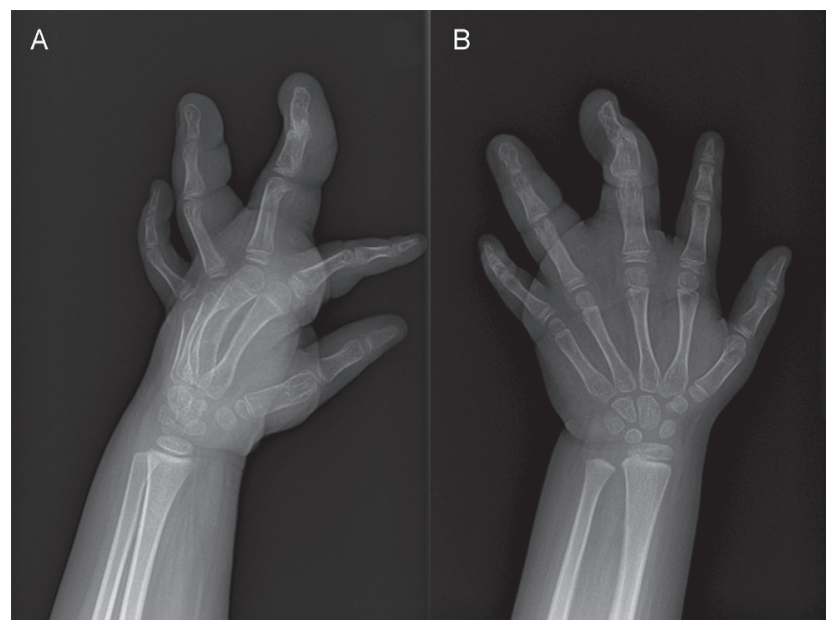

Figure 2. Postoperative 1-year follow-up radiologic features of the patient. The radiographs show enlarged middle and fourth fingers of the patient's left hand (A and B)

Table 1. Medical history timeline

\begin{tabular}{ll}
\hline Time of the investigation & Medical record \\
\hline $07 / 04 / 2015$ & $\begin{array}{l}\text { PE* and X-ray imaging: Large soft tissue } \\
\text { of the middle and ring fingers of the } \\
\text { left hand (Figure 1) }\end{array}$ \\
& $\begin{array}{l}\text { First surgery: Reduction of the left } \\
\text { middle finger with fusion of distal } \\
\text { interphalangeal and proximal interpha- } \\
\text { langeal joints }\end{array}$ \\
& $\begin{array}{l}\text { PE* and X-ray imaging: Enlargement } \\
\text { of subcutaneous, digital nerve, bone, } \\
\text { and tendon of the left middle finger } \\
\text { (Figure 2) } \\
\text { Second surgery: Debulking and correc- } \\
\text { tion osteotomy of the left middle finger }\end{array}$ \\
&
\end{tabular}

$\mathrm{PE}$, physical examination.

middle finger. A surgical complication included a volar pulp flap ischemia. Clinical course of the patient was showed in Table 1.

PCR sequencing of DNA extracted from the affected nerve tissue and cultured dermal fibroblasts isolated from the affected finger revealed the c.3140A $>$ T in PIK3CA (NM_006218.3 and rs121913279), expected to result in an amino acid substitution at position 1047 changing from histidine to leucine (NP_006209.2: p.(H1047L)). This variant was not detected in her peripheral blood leukocytes (Figure 3).

\section{Discussion}

Genetic investigation to identify causative mutations in patients with genetic disorders is important. It will provide

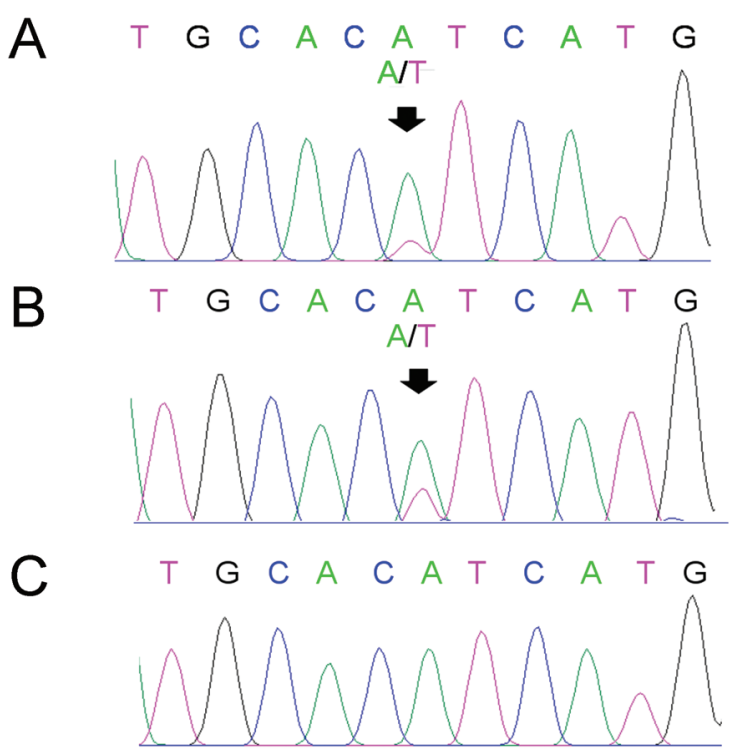

Figure 3. Mutation analysis of the PIK3CA gene. The sequence electropherograms of the PIK3CA from (A) the cultured dermal fibroblasts, (B) the nerve tissues, and (C) peripheral leukocytes. The c.3140A $>T$ mutation (arrows) is found in the dermal fibroblasts and nerve tissue but not peripheral leukocytes

more accurate recurrence risk to the patients and families and may lead to more specific treatment. This patient's parents would like to know the recurrence risk for their next child. The Caucasian patients with isolated macrodactyly have previously been found to have somatic mutations in $P I K 3 C A$ [6-8]. Moreover, other mosaic PIK3CA mutations including p.E542K and p.H1047R have been reported as hotspot mutations in macrodactyly patients of other populations [7]. We therefore aimed to determine whether the proband had a variant in the PIK3CA gene. As the mutation in PIK3CA is somatic, DNA from the affected tissues in addition to leukocytes is required. Fortunately, we received tissues surgically removed from the enlarged finger. PCR sequencing identified p. $(\mathrm{H} 1047 \mathrm{~L})$ of the $P I K 3 C A$ in the proband's nerve and fibroblasts cultured from the affected skin but not in leukocytes. As consistent with previous reports, our study identified the mutation in PIK3CA from both the affected nerve tissue and cultured dermal fibroblasts. The finding suggests that a skin biopsy may be adequate to identify mutations in PIK3CA [9]. This finding confirms that she has the somatic mutation; therefore, the recurrence risk for other family members including future pregnancies is the same as for all other children, which is approximately $0.08 / 10,000$.

The p.H1047L variant in $P I K 3 C A$ is a gain-of-function mutation [10]. PIK3CA is the upstream regulator of the AktmTOR pathway. The p.H1047 affects the kinase domain, 
enhances lipid binding, and results in increased kinase activity [11]. Furthermore, p.H1047L of PIK3CA has been found in colon cancer, breast cancer, and ovarian cancer [12-14]. This leads to a hypothesis that small molecule inhibitors targeting the PI3K-AKT-mTOR pathway may be beneficial to patients with gain-of-function mutations in $P I K 3 C A$ [13]. According to the previous report, treatment with PI3K/ mTOR inhibitor in transgenic mice harboring a hot spot mutation in PIK3CA restored proliferation rate of endothelial cells to normal levels and reduced numbers of senescent cells [15]. Therefore, genetic determination of macrodactyly in our patient may be beneficial not only for more accurate counselling but also for specific treatment in the future.

\section{Conclusion}

As previously identified in other populations, we found the somatic c. $3140 \mathrm{~A}>\mathrm{T}$ p. $(\mathrm{H} 1047 \mathrm{~L})$ variant of $P I K 3 C A$ in a Thai patient with isolated macrodactyly in the affected nerve tissue and cultured dermal fibroblasts, suggesting that a skin biopsy may be adequate to identify variants in PIK3CA.

Author contributions. WD, KS, and VS contributed substantially to the conception and design of this study. WD and PK substantially acquired the data. All the authors analyzed and interpreted it. WD and VS drafted the manuscript. PK and KS critically revised it. All the authors approved the final version submitted for publication and take responsibility for statements made in the published article.

Acknowledgments. We thank the family members for participating in this study. This study was supported by the Thailand Research Fund (DPG6180001) and the Chulalongkorn Academic Advancement into its 2nd Century Project.

Conflict of interest statement. The authors have completed and submitted the International Committee of Medical Journal Editors Uniform Disclosure Form for Potential Conflicts of Interest. None of the authors disclose any conflicts of interest.

\section{References}

[1] Syed A, Sherwani R, Azam Q, Haque F, Akhter K. Congenital macrodactyly: a clinical study. Acta Orthop Belg. 2005; 71:399-404.

[2] da Costa JN, Matias J. Segmental hemihyperplasia-related macrodactyly with congenital renal agenesis: a hand surgeon's point of view. Arch Plast Surg. 2015; 42:655-8.

[3] Iyengar KP, Matar HE, Suraliwala K. Thumb and index finger macrodactyly with first carpometacarpal and scaphotrapezotrapezoidal joints fusion: inevitable consequence? BMJ Case Rep. 2017. doi:10.1136/bcr-2016-218043

[4] Keppler-Noreuil KM, Rios JJ, Parker VE, Semple RK, Lindhurst MJ, Sapp JC, et al. PIK3CA-related overgrowth spectrum (PROS): diagnostic and testing eligibility criteria, differential diagnosis, and evaluation. Am J Med Genet A. 2015; 167A:287-95.

[5] Kotwal PP, Farooque M. Macrodactyly. J Bone Joint Surg Br. 1998; 80:651-3.

[6] Rios JJ, Paria N, Burns DK, Israel BA, Cornelia R, Wise CA, Ezaki M. Somatic gain-of-function mutations in PIK3CA in patients with macrodactyly. Hum Mol Genet. 2013; 22:444-51.

[7] Tripolszki K, Knox R, Parker V, Semple R, Farkas K, Sulak A, et al. Somatic mosaicism of the PIK3CA gene identified in a Hungarian girl with macrodactyly and syndactyly. Eur J Med Genet. 2016; 59:223-6.

[8] Keppler-Noreuil KM, Sapp JC, Lindhurst MJ, Parker VE, Blumhorst $\mathrm{C}$, Darling T, et al. Clinical delineation and natural history of the PIK3CA-related overgrowth spectrum. Am J Med Genet A. 2014; 164A:1713-33.

[9] Wu J, Tian W, Tian G, Sumner K, Hutchinson DT, Ji Y. An investigation of PIK3CA mutations in isolated macrodactyly. J Hand Surg Eur. 2018; 43:756-60.

[10] Kang S, Bader AG, Vogt PK. Phosphatidylinositol 3-kinase mutations identified in human cancer are oncogenic. Proc Natl Acad Sci U S A. 2005; 102:802-7.

[11] Lindhurst MJ, Parker VE, Payne F, Sapp JC, Rudge S, Harris J, et al. Mosaic overgrowth with fibroadipose hyperplasia is caused by somatic activating mutations in PIK3CA. Nat Genet. 2012; 44:928-33.

[12] Campbell IG, Russell SE, Choong DY, Montgomery KG, Ciavarella ML, Hooi CS, et al. Mutation of the PIK3CA gene in ovarian and breast cancer. Cancer Res. 2004; 64:7678-81.

[13] Iacobas I, Burrows PE, Adams DM, Sutton VR, Hollier LH, Chintagumpala MM. Oral rapamycin in the treatment of patients with hamartoma syndromes and PTEN mutation. Pediatr Blood Cancer. 2011; 57:321-3.

[14] Mukohara T. PI3K mutations in breast cancer: prognostic and therapeutic implications. Breast Cancer (Dove Med Press). 2015; 7:111-23.

[15] di Blasio L, Puliafito A, Gagliardi PA, Comunanza V, Somale D, Chiaverina G, et al. PI3K/mTOR inhibition promotes the regression of experimental vascular malformations driven by PIK3CAactivating mutations. Cell Death Dis. 2018; 9:45. 